\title{
Investigating the Effect of Urgency and Modality of Pedestrian Alert Warnings on Driver Acceptance and Performance
}

\author{
David R Large ${ }^{1}$, Hyungil Kim² ${ }^{2}$ Coleman Merenda ${ }^{2}$, Samantha Leong ${ }^{2}$, Catherine Harvey ${ }^{1}$, \\ Gary Burnett ${ }^{1}$, Joseph Gabbard ${ }^{2}$ \\ ${ }^{1}$ Human Factors Research Group, University of Nottingham, Nottingham. UK. \{david.r.large; catherine.harvey; \\ gary.burnett\}@nottingham.ac.uk \\ ${ }^{2}$ COGENT Lab, Virginia Tech, Blacksburg.USA. \{hci.kim; cjm120; sam94; jgabbard\}@vt.edu.
}

\begin{abstract}
Active safety systems have the potential to reduce the risk to pedestrians by warning the driver and/or taking evasive action to reduce the effects of or avoid a collision. However, current systems are limited in the range of scenarios they can address using primary control interventions, and this arguably places more emphasis in some situations on warning the driver so that they can take appropriate action in response to pedestrian hazards. In a counterbalanced experimental design, we varied urgency ('when') based on the time-to-collision (TTC) at which the warning was presented (with associated false-positive alarms, but no false negatives, or 'misses'), and modality ('how') by presenting warnings using audio-only and audio combined with visual alerts presented on a HUD. Results from 24 experienced drivers, who negotiated an urban scenario during twelve 6.0-minute drives in a medium-fidelity driving simulator, showed that all warnings were generally rated 'positively' (using recognised subjective 'acceptance' scales), although acceptance was lower when warnings were delivered at the shortest (2.0s) TTC. In addition, drivers indicated higher confidence in combined audio and visual warnings in all situations. Performance (based on safety margins associated with critical events) varied significantly between warning onset times, with drivers first fixating their gaze on the hazard, taking their foot off the accelerator, applying their foot on the brake, and ultimately bringing the car to a stop further from the pedestrian when warnings were presented at the longest (5.0s) TTC. In addition, drivers applied the brake further from the pedestrian when combined audio and HUD warnings were provided (compared to audio-only), but only at 5.0s TTC. Overall, the study indicates a greater margin of safety associated with the provision of earlier warnings, with no apparent detriment to acceptance, despite relatively high false alarm rates at longer TTCs. Also, that drivers feel more confident with a warning system present, especially when it incorporates auditory and visual elements, even though the visual cue does not necessarily improve hazard localisation or driving performance beyond the advantages offered by auditory alerts alone. Findings are discussed in the context of the design, evaluation and acceptance of active safety systems.
\end{abstract}

\section{Keywords}

Urgency; Modality; Driver Acceptance; Pedestrian Alert Systems; HUD; Vulnerable Road Users; Simulation.

\section{Introduction}

Accidents involving vulnerable road users (VRUs) remain a major issue for road safety, accounting for almost $40 \%$ of road fatalities in Europe, and almost 50\% worldwide [1]. Pedestrians are one of the most vulnerable road user groups, both in terms of the likelihood of being involved in a near-miss or collision, and the potential ramifications should an incident occur. For example, in the US, pedestrians represent 11-13\% of those killed in collisions and are 1.5 times more at risk than vehicle passengers to be fatally injured [2, 3]. Analysis of accident data shows that a high percentage of pedestrian-related incidents occur in urban areas [4], where cars drive at relatively low speeds and the density of pedestrians is likely to be high, with many of these accidents attributed to unexpected or inappropriate pedestrian behaviour, such as crossing the road at the incorrect place or time. For example, in 2013 over $80 \%$ of UK pedestrian road-related fatalities occurred outside of demarcated pedestrian crossings [5]. More recent in-depth accident analysis of European crash databases (including CARE, IGLAD and GIDAS), conducted as part of the PROSPECT project [6], revealed that the most common accident scenario in Europe is situations in which the pedestrian crosses a straight road from the nearside (or offside) with no obstructions. This accounts for the highest proportion of killed or seriously injured pedestrians (KSI) and all pedestrian injuries, and is therefore the designated scenario proposed by the EuroNCAP testing protocol for AEB pedestrian safety systems [7].

The past decade has seen significant progress in active pedestrian safety in passenger cars, in particular due to advances in video and radar technology. This has resulted in the market introduction of first-generation active pedestrian safety systems that can identify at-risk pedestrians and perform autonomous emergency braking (AEB- 
PED) in critical traffic situations. In addition, other primary control interventions, such as steering (and braking jerks or steering wheel vibrations to provide haptic feedback to the driver [8]), are increasingly being integrated within vehicles. Such systems, collectively termed Pedestrian and Cyclist Detection Systems with Emergency Braking (PCDS+EBR), have the potential to mitigate the risk to VRUs by warning drivers of an impending collision and/or taking evasive action autonomously by braking, steering or both, if a collision becomes imminent or if the driver fails to respond. Moreover, evidence suggest that PCDS+EBR systems have the greatest potential (based on current active safety systems) to improve the safety of VRUs, with data indicating a reduction of $7.5 \%$ on all road fatalities and 5.8\% on all road injuries, representing an estimated 2,100 fatalities and over 62,900 injuries saved per year in Europe based on accident trends [9].

However, such systems are non-trivial, requiring the optimisation and integration of situation analysis and collision risk estimation, information and warning concepts, and vehicle control strategies [8]. Moreover, the effectiveness of intervention strategies will vary considerably depending on use-case [8], with first generation systems currently on the market addressing only a limited scope of scenarios [6]. For example, if a driver is turning across the path of a pedestrian or cyclist, and a collision is predicted, the available net braking distance is effectively only $1.57 \mathrm{~m}$ (assuming a typical cornering speed of approximately $20 \mathrm{~km} / \mathrm{h}$ and a deceleration of 9.81 $\mathrm{m} / \mathrm{s}^{2}$ ), and the maximum achievable vehicle displacement from the original driving path will thus be reduced to less than $0.6 \mathrm{~m} \mathrm{[8].} \mathrm{Moreover,} \mathrm{in} \mathrm{this} \mathrm{situation,} \mathrm{braking} \mathrm{and} \mathrm{evasive} \mathrm{steering} \mathrm{can} \mathrm{only} \mathrm{occur} \mathrm{when} \mathrm{the} \mathrm{vehicle} \mathrm{has}$ started to drive in the bend. This arguably places a greater emphasis in this situation on warning the driver so that they can manually instigate appropriate evasive action.

Indeed, as a rule, all systems will attempt to warn the driver before taking any action. Moreover, where possible, warnings will be provided with increasing urgency depending upon the perceived risk level. For example, the PROSPECT system (as realised by Continental [8]), provides two types of VRU collision warning, based on risk level [8]. The first (classified as an 'early warning') is an informative warning to the driver about a potentially dangerous traffic situation involving a VRU, based on a trajectory prediction of VRU and the vehicle but without considering the velocities of both participants, and is therefore necessarily ambiguous. The second type of warning is an imminent acoustic and visual warning, which takes into consideration not only the geometric properties of the predicted trajectories but also the time of potential collision (measured as time-to-collision, TTC, in this case is 1.8 seconds). It is therefore velocity-dependent and assumes that both VRU and the ego vehicle preserve their speeds until the collision occurs. Similarly, the Daimler system provides three warnings (visual and/or auditory), at 4.0 seconds TTC ('early warning'), 2.6 seconds TTC ('warning'), and 1.6 seconds TTC ('urgent warning'), before taking action (i.e. braking at 1.1 seconds TTC).

In addition, ongoing advances in situation analysis, collision risk estimation and intent prediction and modelling (based on improvements in both detection hardware and software algorithms) mean that active safety systems are therefore effectively moving from 'collision mitigation' (with the aim of reducing vehicle speed/kinetic energy to make the impact more survivable) to 'collision avoidance' (aiming to avoid collisions completely). As a consequence, the number of false alarms and/or activations is also expected to increase, in part due to the highly dynamic behaviour of pedestrians, who may change their walking direction and speed, or abruptly start or stop moving at a moment's notice. For example, a pedestrian may approach the roadside, apparently intending to cross, but then stop at the kerb. This could result in a false declaration if the system has already identified the pedestrian as a potential hazard (based on current speeds and trajectories) and highlighted their presence to the driver. Indeed, figures show that first-generation pedestrian safety systems (still on the market) miss approximately $20-30 \%$ of all pedestrians ('false negative'), and issue approximately one false alarm ('false positive'), for every ten presented [10]. While future generations will likely reduce the incidence of false alarms and/or activations, false alarms remain unavoidable in any 'predictive' technology. Moreover, the frequency and occurrence of false alarms and activations will inevitably influence drivers' attitudes towards the system, potentially encouraging them to neglect it, find creative ways to bypass it, or if possible deactivate it completely [11].

The success of such technology is therefore contingent not only on its ability to accurately detect pedestrians, make a precise estimation of their current and future positions with respect to the moving vehicle, and provide timely warnings and/or primary control interventions, but also on driver's attitudes towards it (i.e. their 'acceptance'). On the face of it, these are conflicting goals: by warning drivers sooner, they may have more time and space to respond to warnings, but earlier warnings will be offered at the expense of accuracy, due to both limitations in technologies (range and capability of sensors, accuracy of detection algorithms etc.), as well as idiosyncrasies in the behaviour of pedestrians, and a high number of false declarations will likely detriment acceptance. As such, there is still considerable interest in investigating when and how to present warnings to drivers, in particular to minimise false positive or unnecessary reactions, and improve driver acceptance. 


\section{Acceptance and Urgency}

One of the biggest 'human' challenges in the design of automated vehicle systems is how to keep the driver appraised of the driving situation (i.e. 'in the loop') [12]. Essential to this is the provision of appropriate feedback to the driver about what the system has done, is doing, or is about to do. Norman [13] warned that "proper feedback can make the difference between a pleasurable and successful system and one that frustrates and confuses". In an automotive context, feedback can also be the difference between a safe and an unsafe system, and is particularly important considering the time-criticality of much of the information presented by automated systems [14]. It is also therefore particularly relevant to ensuring an appropriate level of driver acceptance.

The 'acceptance' of in-vehicle driver support systems has been defined as, "the degree to which an individual incorporates the system in his/her driving, or if the system is not available, intends to use it" [15]. The determinants of acceptance are thus complex and derive from various factors, including how individuals use the technology, their understanding of its limits and the context in which it is implemented. Acceptance can also be affected by factors such as the level of trust, confidence, intrusion, or annoyance that users associate with the technology. A common ground in acceptance research is the notion that human behaviour is not primarily determined by objective factors, but by subjective perceptions [16]. This means that acceptance is based on individual attitudes, expectations and experience, obtained during actual use, as well as their subjective evaluation of expected benefits [17]. In fact, it has been shown that the degree of technological innovation has a lesser effect on the acceptance and use of new technological systems than does the amount of personal importance for users [18]. Moreover, for an in-vehicle hazard warning system, in particular, acceptance is considered to be closely related to the driver's perception of risk - not necessarily the actual risk of collision [19]. Drivers are therefore more likely to accept alerts presented in situations that they perceive to be 'alarming' (for example, they have already identified an encroaching pedestrian as a potential threat), even if they would have been able to avoid the incident without the warning [20]. Regardless of the technical capabilities of PCDS, the successful future implementation and integration of such technology will thus depend largely on the degree to which drivers 'accept' the technology, and are willing to place their trust in the system above their own judgement [21].

For a pedestrian alert system, understanding when to present alarms is therefore an important consideration if alarms are presented too early, accuracy may be compromised or the driver may be unaware of the potential risk, thereby negatively impacting acceptance. Moreover, earlier PCDS alerts may be interpreted by the driver as false positive alarms (i.e. the system alerts but no threat is visually detected or acted on), and may consequently degrade drivers' attentiveness to future alarms [22]. On the other hand, alarms that are presented at short TTCs (i.e. 'too late') may be of limited practical use (i.e. the driver may have insufficient time to respond), can startle drivers, and may detriment their reaction and performance during the braking process [23]; late alarms may also annoy drivers, particularly if they are already aware of the hazard and have initiated their own evasive or corrective manoeuvre.

Nevertheless, active safety systems must still accurately detect genuine situations. The concern is that by maximising detection rates, drivers are likely to be flooded with false-alarm warnings, with the inevitable consequence that they will miss or ignore genuine alerts in future safety-critical situations (i.e. the system 'cries wolf') [24]. In contrast, systems designed to minimise false declarations may miss genuine safety-critical situations, and may provide insufficient exposure to allow drivers to calibrate their trust in system [25]. Moreover, in the absence of false alarms, genuine alerts may be so rare as to be utterly unfamiliar and consequently drivers' reactions will be unpredictable (i.e. efficient recall of how to react depends on the frequency of use [26]).

\section{Feedback and Modality}

A further consideration is how to present warnings to drivers. Visual feedback is typically presented to the driver using either of two methods: traditional displays or ambient displays. Traditional displays are defined as screens in the instrument cluster, centre console or dashboard and usually require foveal vision to attend to the presented information. Traditional visual displays usually have associated issues of distraction as they draw the driver's attention away from the road scene - the larger the eccentricity of display location from forward view, the longer the eyes-off road time. In contrast, ambient displays aim to utilise peripheral vision by displaying feedback (e.g. lighting effects) in the A-pillars, doors, dashboard, steering wheel, and interior and exterior mirrors (see for examples: $[8,27,28])$. Ambient displays are much more limited in the bandwidth of information content compared with traditional displays, but offer a benefit in that they overcome some of the issues of distraction, requiring only peripheral attention.

In addition, visual information can be presented 'externally' to the vehicle, via a Head-Up Display (HUD). HUD technology allows visual information to be presented using a transparent display located in the driver's normal field of view. Consequently, feedback appears to exist 'in the world' rather than inside the vehicle. By presenting information directly within the forward-facing line of sight (i.e. on or 'through' the windscreen), drivers can access that information without lowering or diverting their gaze away from the road scene, thus avoiding the 
apparent gaps in visual attention ('eyes-off-road') associated with using more traditional 'head-down' presentations. Moreover, drivers are not required to constantly re-accommodate their focal length between the distant road scene and nearby in-vehicle displays - a particularly important advantage for older drivers who experience problems with accommodating their vision to near objects [29]. Additionally, information presented via a HUD can be spatially linked to and augment the information in the real world to which it relates ('conformal') [30]. In the context of a warning system, conformal visual feedback (using a HUD) has been shown to help drivers localize threats earlier [31, 32]. Additionally, using a HUD can command attention more strongly during the decision-making phase, and can help mitigate other functional weaknesses (such as poorly designed controls or a suboptimal field of view of the road) that may exist between the driver and human-machine interface (HMI) [33, $34,35]$.

In contrast, auditory alerts that can be heard by the driver can enhance the recognition of warnings, afford better semantic association, and improve drivers' responses, but are typically unable to visually guide attention towards the threat [36]. However, combining auditory warnings with visual information to provide 'multimodal' feedback can reduce human reaction times [37, 38], and can be particularly effective for reorienting spatial attention [39]. Consequently, several novel HMI concepts to inform or warn the driver have been proposed (see examples in [8]); these tend to incorporate both acoustic warnings and visual elements, although, it has also been suggested that in some cases it may be more appropriate to provide unimodal feedback (for example, auditory only) assuming the information is understandable and able to capture attention in the chosen modality.

\section{Overview and Aim of Study}

Aiming to take all of these factors into consideration, the study explored the effect of the urgency and modality of alert warnings associated with potential pedestrian hazards detected by an active safety system. To achieve this, urgency was varied based on the TTC at which the warning was presented (calculated using the existing speeds and trajectories of car and pedestrian). Moreover, a linear relationship between TTC and false alarm rate was applied, with a higher proportion of false alarms associated with warnings delivered at longer TTCs. This approach is based on previous literature indicating that the probability of making a correct judgement of whether a pedestrian will cross or not in this scenario reduces at an approximate linear rate with increasing TTC [40]. The intention in taking this approach was primarily to reflect the higher false alarm rates based on the provision of earlier warnings, i.e. the system appears less 'reliable' at longer TTCs - as would be expected with such technology, and is not intended as a validation of this assumption.

For each level of urgency, either no warning, an audio alert, or an audio alert combined with a visual warning projected via a HUD were explored (to investigate modality). Driver acceptance was subsequently determined using subjective ratings of trust, confidence, annoyance, desirability, predictability, reliance and risk (based on existing acceptance/usability assessment protocols [41]). In addition, objective performance was calculated based on safety margins associated with critical primary control events and drivers' visual behaviour (i.e. the TTC/headway from the pedestrian at which drivers first located and fixated their gaze on the pedestrian hazard, and the TTC/headway at which they took their foot off the accelerator, applied the brake, and ultimately brought the car to a stop).

\section{Method}

Twenty-four drivers took part in the study: 15 male, 9 female, with ages ranging from 19 to 55 years, representative of an active UK driving population. All participants were required to have at least 2 years of driving experience (mean: 10.7 years). Participants were self-selecting volunteers who responded to advertisements placed around the University of Nottingham campus and were reimbursed with $£ 15$ (GBP) of shopping vouchers as compensation for their time. All participants provided written informed consent.

The study took place in a medium-fidelity, fixed-based driving simulator at the University of Nottingham (Figure 1). The simulator comprises an Audi TT car located within a curved screen, affording 270 degrees forward and side image of the driving scene via three overhead HD projectors, together with rear and side mirror displays. A Thrustmaster 500RS force feedback steering wheel and pedal set are integrated with the existing Audi steering wheel and pedals, and the dashboard is created using a bespoke Java application and presented on a 7-inch LCD screen, which replaces the original instrument cluster. 


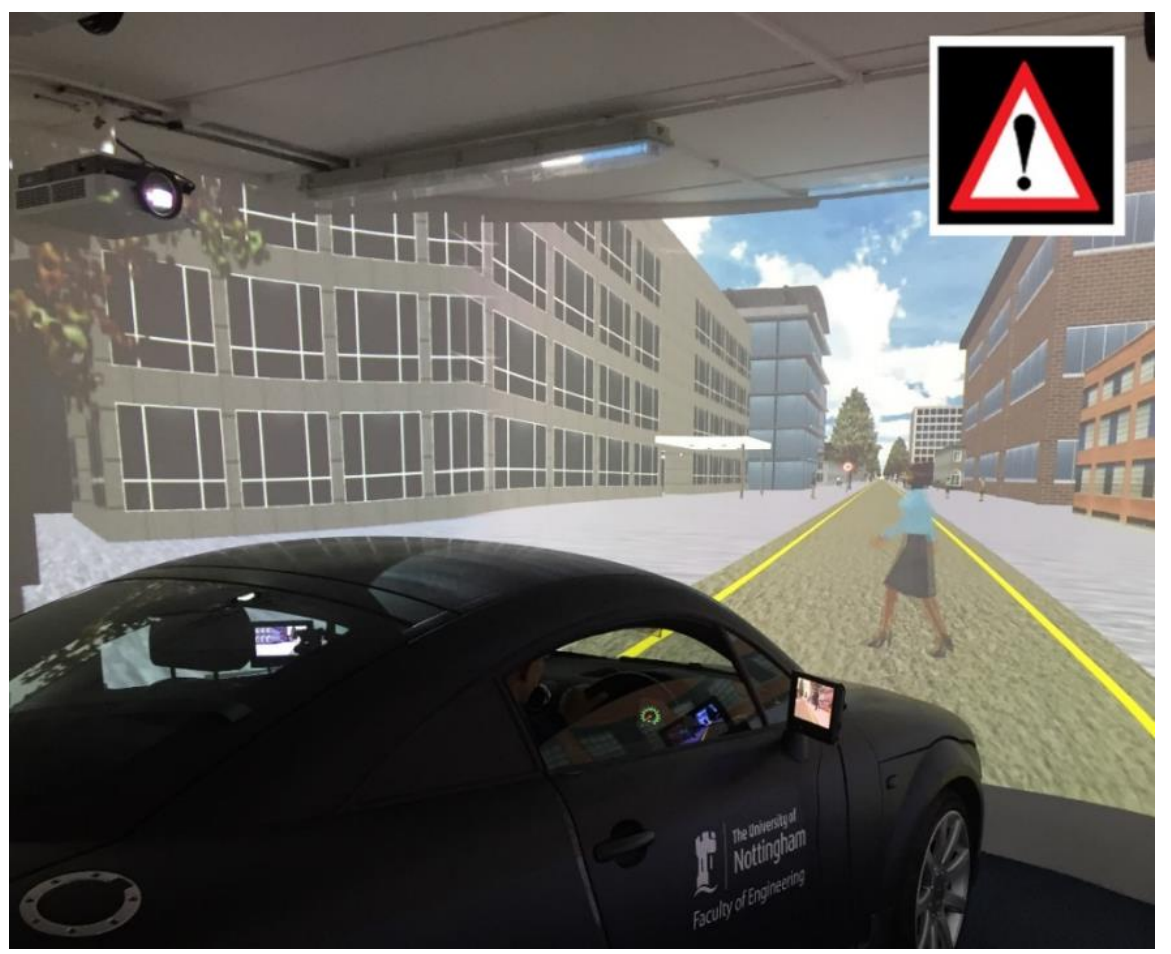

Figure 1: Driving simulator showing urban high-street scenario and pedestrian crossing road ahead, with visual alert icon that was presented on the HUD (inset).

The driving scenario was created using STISIM Drive (version 3) to replicate a straight, single-carriageway road traversing through a built-up urban environment, absent of demarcated pedestrian crossings. Pavements ('side-walks') were populated with approximately 5-10 pedestrians every fifty feet, who appeared in a random distribution, and walked forward and backward in parallel with the road, or stood facing the roadway but not moving. These pedestrians were included in the scenario to present a realistic, 'visually-cluttered' urban backdrop and to ensure that identifying the 'hazard' (i.e. the rogue pedestrian/s who walked towards the roadway) was nontrivial.

In addition to the 'background' pedestrians, a further five pedestrians were placed at random distances into the scenario, and approached the roadside as if intending to cross the road. Each of the five 'hazard' pedestrians were initially standing motionless approximately one metre from the roadway (corresponding with the centre of the 'sidewalk') and facing the road. As such, they were 'always' visible to the driver (i.e. they did not move into view from behind an obstruction as part of the crossing manoeuvre, or suddenly materialise as the driver approached). They walked at a steady pace to ensure that they would reach the centre of the roadway at the same time as the car (assuming they continued to do so). To achieve this, the total walking distance did not vary between conditions. Instead, walking speed was varied (from .5 to $3 \mathrm{mph}$, i.e. slightly slower than a brisk walk, typically $4 \mathrm{mph}$ ) to ensure that vehicle and pedestrian trajectory would intersect (assuming neither party deviated from their current trajectory or speed).

Depending on the scenario (see Table 1), a proportion of the 'hazard' pedestrians then continued to cross the road (representing the most common pedestrian accident use-case [6]), while the remainder stopped at the roadside. All five of these pedestrians were identified by the PCDS system, thereby giving rise to 'false positive alarms' in the latter situation (i.e. the system accurately detected the presence of a pedestrian but falsely predicted their intention to enter the roadway). Participants in the study were instructed to drive along the road and maintain a constant speed of $25 \mathrm{mph}(40 \mathrm{kph})$. No specific instructions were provided to drivers regarding how they should respond to warnings, other than to behave 'naturally'. They were not specifically told that some pedestrians might attempt to cross the roadway.

Warnings were presented at four different levels of urgency, as defined by the TTC, with the number of accurately detected pedestrians (i.e. those who continued to cross the road) decreasing with increasing TTC, thus simulating an increasing false alarm rate. This approach is predicated on the fact that the probability of making a correct judgement of whether a pedestrian will cross or not (as they approach the roadside) reduces at an approximately linear rate with increasing TTC [40]. The TTCs (and associated false alarm rates) were thus selected to conform approximately with the performance of current state-of-the-art [8]. TTCs therefore ranged from 5.0seconds (with a false alarm rate of $80 \%$, i.e. 4 out of 5 pedestrians approached, but ultimately did not cross the road) to 2.0 -seconds (with a false alarm rate of $20 \%$ or 1 in 5 ). 
Warnings were presented within the vehicle as a static visual alert icon and/auditory icon presented at $75 \mathrm{~dB}$ (based on design guidelines [42]). A generic high-contrast visual alert icon was selected to aid ease of recognition and saliency to visual attention (Figure 1). The icon was presented for a period of up to 4.0 -seconds, based on design recommendations [43]. Visual warnings were provided using a Pioneer laser-scanning head-up display (HUD). In addition, both visual and auditory warnings were spatially congruent (i.e. presented to correspond with the side of the road from which the pedestrian approached), with the aim of directing and localising the driver's gaze to the target pedestrian.

In addition to completing the drive with auditory and auditory/visual warnings, participants also repeated it for each false alarm rate with no warnings. Thus, participants completed 12 drives (Table 1). During 'No Warning' scenarios, the urgency levels indicate the equivalent distance at which a warning (had it been presented) would have generated a false alarm rate in line with our study design, and are used for comparative analysis between the three conditions. Conditions were counterbalanced, but participants completed all conditions for each warning type (and 'no warnings') together.

Table 1: Twelve scenarios completed by all participants, showing TTCs and false alarm rates (i.e. number of pedestrians who did not step into the road but were nevertheless highlighted by the PCDS).

\begin{tabular}{ccccc}
\hline $\begin{array}{c}\text { Urgency } \\
\text { Level }\end{array}$ & $\begin{array}{c}\text { TTC } \\
\text { (when warning } \\
\text { presented) }\end{array}$ & $\begin{array}{c}\text { Audio Only } \\
\text { (4 scenarios) }\end{array}$ & $\begin{array}{c}\text { Combined } \\
\text { (Audio+HUD) } \\
\text { (4 scenarios) }\end{array}$ & $\begin{array}{c}\text { No } \\
\text { Warning } \\
\text { (4 scenarios) }\end{array}$ \\
\hline L1 & $2.0-\mathrm{sec}$ & $20 \%(1$ in 5) & $20 \%(1$ in 5) & $20 \%(1$ in 5) \\
L2 & $3.0-\mathrm{sec}$ & $40 \%(2$ in 5) & $40 \%(2$ in 5) & $40 \%(2$ in 5) \\
L3 & $4.0-\mathrm{sec}$ & $60 \%(3$ in 5) & $60 \%(3$ in 5) & $60 \%(3$ in 5) \\
L4 & $5.0-\mathrm{sec}$ & $80 \%(4$ in 5) & $80 \%(4$ in 5) & $80 \%(4$ in 5) \\
\hline
\end{tabular}

\section{Timeline of Events}

During each scenario, participants drove for approximately 45 seconds before encountering any hazards. Thereafter, they were presented with the first deviant pedestrian (who walked towards the roadside as if intending to cross) randomly within the next $300 \mathrm{~m}$, approximately (i.e. $1000 \mathrm{ft}$ ). No events occurred within the subsequent $300 \mathrm{~m}$, to ensure that participants were not presented with back-to-back hazards. Participants then experienced the second pedestrian crossing event in the following $300 \mathrm{~m}$, and so on. This effectively resulted in the driver experiencing about 30-45 seconds of uninterrupted driving, followed by the next 30-45 seconds in which an event occurred. This pattern was repeated five times, but because of the random variability within each time window, it was not possible to predict events.

After each pedestrian event (with associated warning intervention, where appropriate), participants resumed driving only after they felt sure that it was safe to continue (i.e. when they could confirm that the pedestrian had either stopped at the roadside, or had finished crossing the road). Each drive therefore lasted approximately 6-8 minutes. Overall, the study took approximately $1 \frac{1}{2} 2$ hours for each participant.

\section{Measures}

Acceptance was determined using subjective measures, i.e. by asking participants to rate their attitudes towards the PCDS using recognised Likert scales (where $0=$ 'not at all' and $10=$ 'completely'), immediately following each of the twelve drives (with each drive corresponding to a specific warning type and time, i.e. TTC). Scales were taken from existing acceptance/usability assessment protocols [41], with ratings captured by inviting responses to seven questions exploring the constructs of trust, confidence, annoyance, desirability, predictability, reliance and risk (which collectively were interpreted as 'acceptance'):

1. Overall, how much do you trust the system? (Trust)

2. How confident are you that the system will be able to cope with all situations in the future? (Confidence)

3. How annoying was the system? (Annoyance)

4. How likely would you be to use the system if it was available in your own car? (Desirability)

5. To what extent can the system's behaviour be predicted from moment to moment? (Predictability)

6. To what extent can you count on the system to do its job? (Reliance)

7. To what extent will the system's actions have a harmful or injurious outcome? (Risk) 
Performance was determined based on data captured by the simulator software and visual behaviour from SensoMotoric Instruments Natural Gaze eye-tracking glasses, which were worn by participants throughout the study. A range of objective performance measures were subsequently defined, based on the headway/TTC at which critical events occurred (effectively, surrogate safety margins) (Table 2). These were determined by synchronising the eye-tracking data with the performance data captured from the driving simulator (for gaze-related measures), or directly from the simulator data for primary control actions (foot off accelerator, foot on brake) and vehicle behaviour (car stopped). In addition, reaction times and stopping distances were captured (using a similar approach), and these have been reported in Large et al. [44] and Merenda et al. [21]; the reader is advised to refer to these papers for further information on these measures.

Table 2: Subjective acceptance and objective performance measures analysed

\begin{tabular}{ll}
$\begin{array}{c}\text { Subjective Ratings } \\
\text { ('Acceptance') }\end{array}$ & \multicolumn{1}{c}{$\begin{array}{c}\text { Objective Performance Measures } \\
\text { ('Surrogate Safety Margins') }\end{array}$} \\
\hline Trust & TTC $(\mathrm{s}) / \mathrm{Headway}(\mathrm{m})$ @ gaze on PED \\
Confidence & $\mathrm{TTC}(\mathrm{s}) / \mathrm{Headway}(\mathrm{m})$ @ foot off accelerator \\
Annoyance & $\mathrm{TTC}(\mathrm{s}) / \mathrm{Headway}(\mathrm{m})$ @ foot on brake \\
Desirability & Headway $(\mathrm{m})$ @ car stopped \\
Predictability & \\
Reliance & \\
Risk & \\
\hline
\end{tabular}

\section{Results and Analysis}

\section{Data Analysis}

To identify main and interaction effects of Urgency (measured by TTC $=\{2,3,4,5\}$ seconds) and Modality of warning $(\mathrm{HMI}=\{$ no, Audio, Audio+HUD $\})$ on driving performance and driver acceptance, we performed twoway, repeated-measures ANOVAs. Prior to this, data were tested for normality using a Shapiro-Wilk normality test. For those data that were not normally distributed, ANOVAs were performed on log-transformed values, which were subsequently back-transformed to provide summary statistics (e.g. means and standard error of means) in their original units for reporting. In situations where significant effects were revealed, we conducted post-hoc contrast tests for planned comparisons among experimental conditions to investigate any interaction effects, applying Tukey's adjustment to account for multiple comparisons. All data analysis was conducted using the statistical software program R (version 3.2.2), and the 'Ime4' package for the mixed effects ANOVA, considering Urgency and Modality of warning as fixed effect factors, and Participant as a random effect factor.

\section{Subjective Ratings}

Descriptive statistics and main effects/interactions for subjective ratings are shown in Table 3 and Table 4. In addition, ratings are shown as box plots with $95 \%$ confidence intervals (Figure 2). There were significant main effects of Urgency evident for Trust $(\mathrm{p}<.01)$, Confidence $(p<.01)$, Desirability $(p<.05)$, Predictability $(p<.001)$, Reliance $(p<.01)$ and Risk $(p<.05)$. Post-hoc tests indicated differences in perceived Trust, Confidence, Desirability (at $p<.10$ ), Predictability and Reliance between L1 and \{L2, L3 $\}$ and between L1 and L3 for Risk. In addition, there were significant differences between L1 and L4 for Predictability and Risk. Although overall, ratings were statistically 'worse' at L1 (lower for positive terms and higher for negative terms), all subjective ratings were generally positive in all situations (above 6 for positive terms, and below 5 for negative terms) (Figure 2). Even so, there were also significant effects of Modality for Confidence, with post-hoc tests showing that differences existed between audio-only (W1) and audio+HUD warnings (W2), across all levels of Urgency, with higher ratings for combined warnings. 
(a) Trust

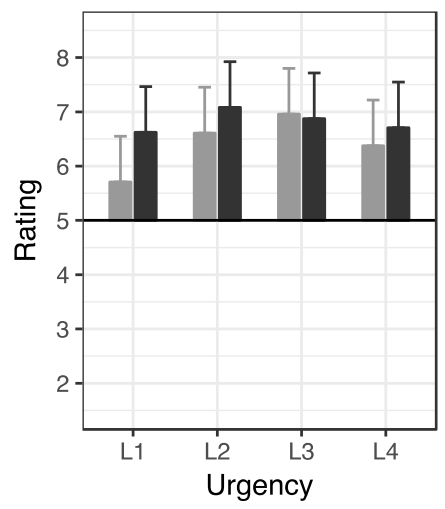

(e) Predictability

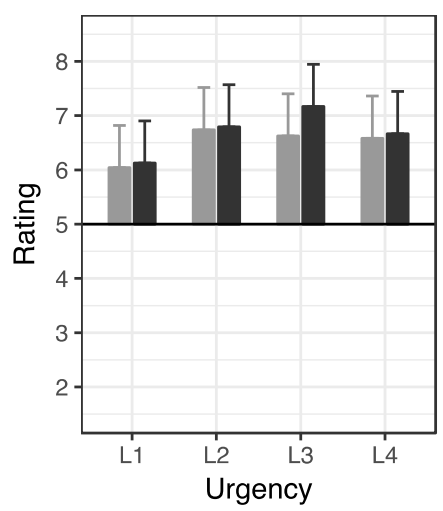

(b) Confidence

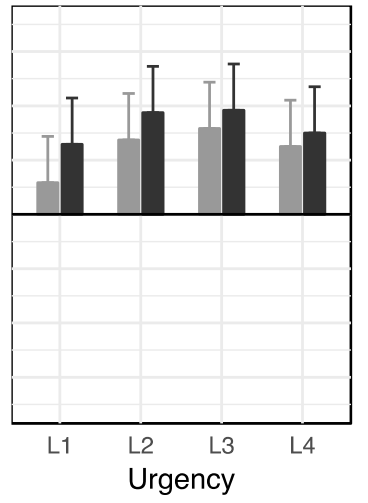

(f) Reliance

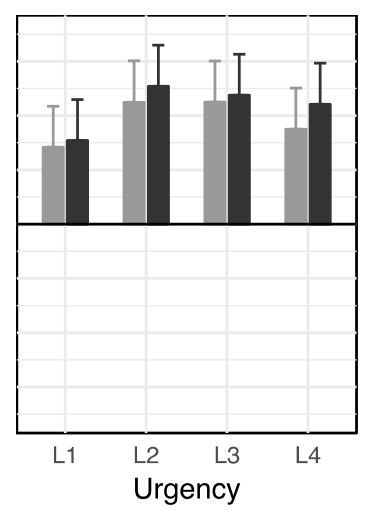

(c) Annoyance

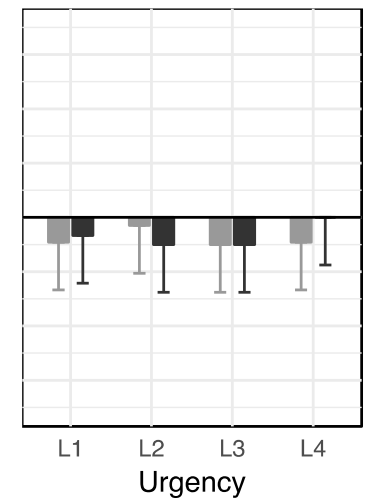

(g) Risk

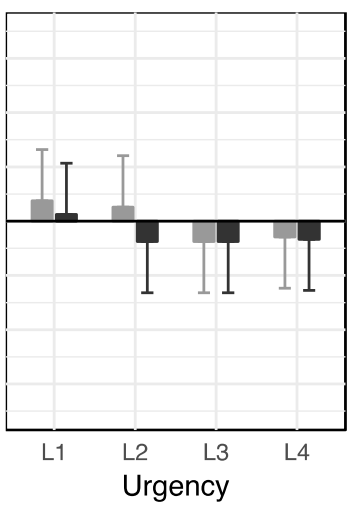

(d) Desirability

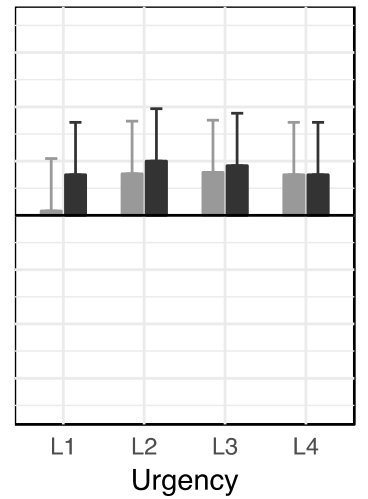

HMI

w1: Audio

w2: Audio + HUD

Figure 2: Drivers' subjective ratings by urgency and modality, where $0=$ 'not at all' and $10=$ 'completely', with $95 \%$ confidence intervals. 
Table 3: Drivers' acceptance, reported by subjective ratings, showing means (standard error of the means, corrected for within-subject variability) in each experimental condition

\begin{tabular}{|c|c|c|c|c|c|c|c|c|}
\hline $\begin{array}{l}\text { Modality } \\
\text { (HMI) }\end{array}$ & Urgency & Trust & Confidence & Annoyance & Desirability & Predictability & Reliance & Risk \\
\hline Audio & $\mathrm{L} 1$ & $5.7(0.42)$ & $5.6(0.42)$ & $4.5(0.43)$ & $5.1(0.47)$ & $6.0(0.39)$ & $6.4(0.38)$ & $5.4(0.47)$ \\
\hline Audio+HUD & L1 & $6.6(0.42)$ & $6.3(0.42)$ & $4.7(0.43)$ & $5.8(0.48)$ & $6.1(0.39)$ & $6.5(0.38)$ & $5.1(0.47)$ \\
\hline Audio & L2 & $6.6(0.42)$ & $6.4(0.43)$ & $4.9(0.43)$ & $5.8(0.48)$ & $6.7(0.39)$ & $7.2(0.38)$ & $5.3(0.47)$ \\
\hline Audio+HUD & $\mathrm{L} 2$ & $7.1(0.42)$ & $6.9(0.42)$ & $4.5(0.43)$ & $6.0(0.48)$ & $6.8(0.39)$ & $7.5(0.38)$ & $4.6(0.47)$ \\
\hline Audio & L3 & $7.0(0.42)$ & $6.6(0.42)$ & $4.5(0.43)$ & $5.8(0.47)$ & $6.6(0.39)$ & $7.3(0.38)$ & $4.6(0.47)$ \\
\hline Audio+HUD & L3 & $6.9(0.42)$ & $6.9(0.42)$ & $4.5(0.43)$ & $5.9(0.47)$ & $7.2(0.39)$ & $7.4(0.38)$ & $4.6(0.47)$ \\
\hline Audio & $\mathrm{L} 4$ & $6.4(0.42)$ & $6.3(0.42)$ & $4.5(0.43)$ & $5.8(0.48)$ & $6.6(0.39)$ & $6.8(0.38)$ & $4.7(0.47)$ \\
\hline Audio+HUD & $\mathrm{L} 4$ & $6.7(0.42)$ & $6.5(0.42)$ & $5.0(0.43)$ & $5.8(0.48)$ & $6.7(0.39)$ & $7.2(0.38)$ & $4.7(0.47)$ \\
\hline
\end{tabular}

Table 4: Main and interaction effects of the urgency and modality of PCDS on driver subjective ratings $(* p<0.05, * * p<0.01, * * * p<0.001)$

\begin{tabular}{|c|c|c|c|c|c|c|c|}
\hline Effect & Trust & Confidence & Annoyance & Desirability & Predictability & Reliance & Risk \\
\hline Urgency & $\mathrm{F}(3,137.1)=5.111^{* *}$ & $\mathrm{~F}(3,69.0)=4.148^{* *}$ & $\mathrm{~F}(3,67.9)=0.660$ & $\mathrm{~F}(3,137.1)=2.843^{*}$ & $\mathrm{~F}(3,68.1)=6.021^{* * *}$ & $\mathrm{~F}(3,68.8)=5.534^{* *}$ & $\mathrm{~F}(3,67.8)=3.623^{*}$ \\
\hline Modality & $\mathrm{F}(1,22.7)=2.586$ & $\mathrm{~F}(1,23.0)=4.590^{*}$ & $\mathrm{~F}(1,22.9)=0.082$ & $\mathrm{~F}(1,23.0)=1.036$ & $\mathrm{~F}(1,22.9)=0.471$ & $\mathrm{~F}(1,22.9)=0.932$ & $\mathrm{~F}(1,21.9)=1.069$ \\
\hline Urgency*Modality & $\mathrm{F}(3,137.1)=1.868$ & $F(3,69.0)=0.535$ & $\mathrm{~F}(3,68.6)=1.703$ & $\mathrm{~F}(3,137.1)=1.308$ & $\mathrm{~F}(3,68.5)=1.115$ & $\mathrm{~F}(3,68.8)=0.389$ & $\mathrm{~F}(3,69.2)=0.961$ \\
\hline
\end{tabular}




\section{Driving Performance (Headway and TTC)}

Descriptive statistics for driving performance measures with main effects and significant interactions are shown in Table 5 and Table 6, respectively. It is worth highlighting that due to the nature of the driving environment (i.e. a single lane carriageway), steering interventions were not practically possible, and therefore drivers reacted to the pedestrian threats by braking or otherwise reducing the speed of their vehicle.

For TTC @ Gaze on Pedestrian, there was a main effect of Urgency $(\mathrm{F}(3,44.6)=89.94, p<.001)$. Post-hoc tests show significant differences between all levels of Urgency (all $p<0.001$ ), with TTC @ Gaze on Pedestrian increasing as TTC increased. There were no significant main effects for Modality $(\mathrm{F}(2,36.9)=0.50, \mathrm{p}=0.608)$, and no significant interaction between Urgency and Modality $(F(6,82.3)=1.73, \mathrm{p}=0.125)$.

Table 5: Driving performance measures, reported by surrogate safety metrics - means (standard error of the means, corrected for within-subject variability) in each experimental condition.

\begin{tabular}{|c|c|c|c|c|c|}
\hline $\begin{array}{c}\text { Modality } \\
\text { (HMI) }\end{array}$ & Urgency & $\begin{array}{c}\text { TTC (s) } \\
\text { @ Gaze on } \\
\text { Pedestrian }\end{array}$ & $\begin{array}{c}\text { TTC (s) } \\
\text { @ Foot off } \\
\text { Accelerator }\end{array}$ & $\begin{array}{c}\text { TTC (s) } \\
\text { @ Foot on } \\
\text { Brake }\end{array}$ & $\begin{array}{c}\text { Headway (m) } \\
\text { @ Car Stopped }\end{array}$ \\
\hline None & L1 & $1.59(0.12)$ & $1.42(0.02)$ & $1.05(0.04)$ & $18.44(1.56)$ \\
\hline Audio & $\mathrm{L} 1$ & $1.43(0.11)$ & $1.46(0.02)$ & $1.11(0.04)$ & 20.49 (1.67) \\
\hline Audio+HUD & $\mathrm{L} 1$ & $1.39(0.09)$ & $1.47(0.02)$ & $1.12(0.04)$ & $21.03(1.71)$ \\
\hline None & $\mathrm{L} 2$ & $2.31(0.14)$ & $2.26(0.02)$ & $1.56(0.05)$ & $37.41(3.08)$ \\
\hline Audio & $\mathrm{L} 2$ & $2.44(0.14)$ & $2.37(0.03)$ & $1.73(0.05)$ & $44.39(3.61)$ \\
\hline Audio+HUD & $\mathrm{L} 2$ & $2.36(0.13)$ & $2.42(0.03)$ & $1.71(0.05)$ & $43.65(3.55)$ \\
\hline None & L3 & $2.84(0.17)$ & $3.05(0.03)$ & $1.87(0.06)$ & $41.57(3.47)$ \\
\hline Audio & L3 & $3.23(0.19)$ & $3.28(0.03)$ & $2.22(0.07)$ & $57.48(4.68)$ \\
\hline Audio+HUD & L3 & $3.07(0.16)$ & $3.33(0.04)$ & $2.22(0.07)$ & $54.10(4.40)$ \\
\hline None & $\mathrm{L} 4$ & $3.12(0.18)$ & $3.42(0.04)$ & $2.28(0.07)$ & $42.50(3.50)$ \\
\hline Audio & L4 & $3.44(0.19)$ & $4.23(0.05)$ & $2.85(0.09)$ & $64.69(5.41)$ \\
\hline Audio+HUD & L4 & $3.74(0.20)$ & $4.33(0.05)$ & $3.14(0.10)$ & $64.20(5.29)$ \\
\hline
\end{tabular}

Table 6: Main and interaction effects of the urgency and modality of PCDS on surrogate safety metrics $(\# p<0.1, * p<0.05, * * p<0.01, * * * p<0.001)$.

\begin{tabular}{ccccc}
\hline Effect & $\begin{array}{c}\text { TTC (s) } \\
\text { @ Gaze on Ped. }\end{array}$ & $\begin{array}{c}\text { TTC (s) } \\
\text { @ Foot off Acc. }\end{array}$ & $\begin{array}{c}\text { TTC (s) } \\
\text { @ Foot on Brake }\end{array}$ & $\begin{array}{c}\text { Headway (m) } \\
\text { @ Car Stopped }\end{array}$ \\
\hline Urgency & $\mathrm{F}(3,44.6)=89.94 * * * *$ & $\mathrm{~F}(3,67.4)=5037.10^{* * *}$ & $\mathrm{~F}(3,67.6)=427.09^{* * *}$ & $\mathrm{~F}(3,45.8)=117.97 * * *$ \\
Modality & $\mathrm{F}(2,36.9)=0.50$ & $\mathrm{~F}(2,44.1)=103.84 * * *$ & $\mathrm{~F}(2,45.8)=41.73^{* * *}$ & $\mathrm{~F}(2,44.1)=15.12^{* * *}$ \\
Urgency*Modality & $\mathrm{F}(6,82.3)=1.73$ & $\mathrm{~F}(6,132.8)=33.03^{* * *}$ & $\mathrm{~F}(6,135.8)=5.79^{* * *}$ & $\mathrm{~F}(6,106.4)=2.00^{\#}$ \\
\hline
\end{tabular}


For TTC @ Foot off Accelerator, there were main effects for Urgency $(\mathrm{F}(3,67.4)=5037.10, p<.001)$ and Modality $(\mathrm{F}(2,44.1)=103.84, p<.001)$, and an interaction between Urgency and Modality $(\mathrm{F}(6,132.8)=33.03$, $p<.001$ ). Post-hoc tests (Table 7) show significant differences between all levels of Urgency for all warning types, indicating that drivers took their foot off the accelerator further from the pedestrian when warnings were provided at longer TTCs. In addition, there were significant differences between warning types and Urgency showing that when warnings were provided at the longest TTC, drivers' initial response (i.e. taking their foot off the accelerator) occurred further from the pedestrian than when no warning was provided. Moreover, differences between having a warning and not having a warning became more pronounced as TTC increased (Figure 3).

Table 7: Significant post-hoc tests for TTC @ Foot off Accelerator between warning types (W0=None, W1=Audio, W2=Audio+HUD)

\begin{tabular}{ccc}
\hline Contrast & Urgency & Statistics \\
\hline w0 - w2 & L1 & $\mathrm{t}(168.9)=-2.55, \mathrm{p}=0.031$ \\
w0 - w1 & L2 & $\mathrm{t}(163.6)=-3.63, \mathrm{p}=0.001$ \\
w0 - w2 & L2 & $\mathrm{t}(163.6)=-5.34, \mathrm{p}<0.001$ \\
w0 - w1 & L3 & $\mathrm{t}(166.2)=-5.50, \mathrm{p}<0.001$ \\
w0 - w2 & L3 & $\mathrm{t}(166.2)=-6.77, \mathrm{p}<0.001$ \\
w0 - w1 & L4 & $\mathrm{t}(165.1)=-15.92, \mathrm{p}<0.001$ \\
w0 - w2 & L4 & $\mathrm{t}(166.9)=-17.78, \mathrm{p}<0.001$ \\
\hline
\end{tabular}

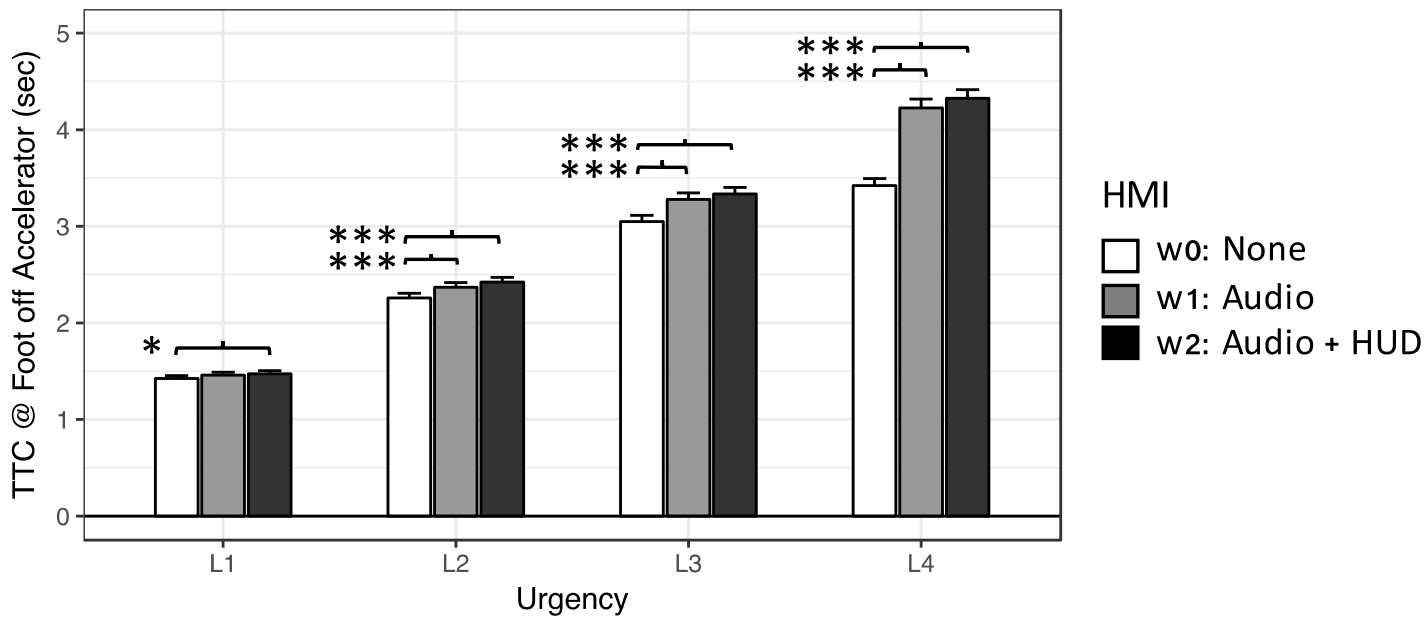

Figure 3: TTC @ Foot off Accelerator by urgency and modality, with 95\% confidence intervals $(* p<0.05, * * p<0.01, * * * p<0.001)$

ForTTC @ Foot on Brake, there were main effects for Urgency $(\mathrm{F}(3,67.6)=427.09, p<.001)$ and Modality $(\mathrm{F}(2,45.8)=41.73, p<.001)$, and an interaction between Urgency and Modality $(\mathrm{F}(6,135.8)=5.79, p<.001)$. Post-hoc tests (Table 8) show significant differences between all levels of Urgency for all warning types $(p<.001)$, although there were only significant differences between warning types (i.e. between w1 and w2) at TTC $=5$ seconds $\left(p_{\max }=.024\right)$. Again, differences between having a warning and not having a warning became more pronounced as TTC increased (Figure 4). 
Table 8: Significant post-hoc tests for TTC @ Foot on Brake between warning types (W0=None, W1=Audio, W2=Audio+HUD)

\begin{tabular}{ccc}
\hline Contrast & Urgency & Statistics \\
\hline w0 - w1 & L2 & $\mathrm{t}(173.7)=-3.02, \mathrm{p}=0.008$ \\
w0 - w2 & L2 & $\mathrm{t}(173.7)=-2.70, \mathrm{p}=0.021$ \\
w0 - w1 & L3 & $\mathrm{t}(175.6)=-4.83, \mathrm{p}<0.001$ \\
w0 - w2 & L3 & $\mathrm{t}(175.6)=-4.83, \mathrm{p}<0.001$ \\
w0 - w1 & L4 & $\mathrm{t}(173.9)=-6.19, \mathrm{p}<0.001$ \\
w0 - w2 & L4 & $\mathrm{t}(176.8)=-8.93, \mathrm{p}<0.001$ \\
w1 - w2 & L4 & $\mathrm{t}(178.9)=-2.65, \mathrm{p}=0.024$ \\
\hline
\end{tabular}

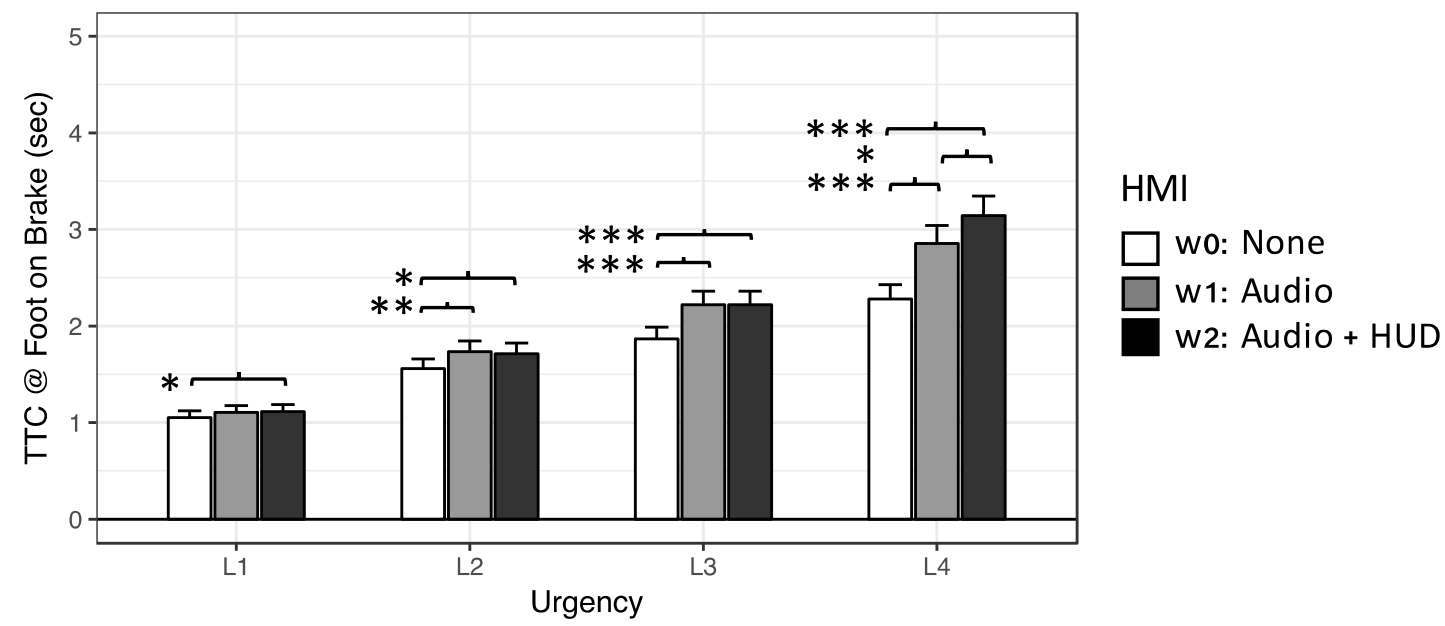

Figure 4: TTC @ Foot on Brake by urgency and modality, with 95\% confidence intervals $(* p<0.05, * * p<0.01, * * * p<0.001)$

For Headway @ Car Stopped, there were main effects for Urgency $(\mathrm{F}(3,45.8)=117.97, p<.001)$ and Modality $(\mathrm{F}(2,44.1)=15.12, p<.001)$, and an interaction between Urgency and Modality $(\mathrm{F}(6,135.8)=5.79, p=.072)$ (significant at $p<.10$ ). Post-hoc tests (Table 9) show significant differences between L1 and \{L2, L3,L 4\} for all warning types, between L2 and $\{\mathrm{L} 3, \mathrm{~L} 4\}$ for audio-only, and between L2 and L4 for audio+HUD warnings (all $p$ $<.001$ ). In addition, significant differences were evident at L3 and L4 between no-warnings and audio-only, audio+HUD $\}\left(p_{\max }=.007\right)$. There were no significant differences between warning types (w1 and $\mathrm{w} 2$ ) at any levels of Urgency. It was also evident, as before, that differences between having a warning and not having a warning became more pronounced as TTC increased (Figure 5). 
Table 9: Significant post-hoc tests for Headway @ Car Stopped (W0=None, W1=Audio, W2=Audio+HUD) $\left({ }^{\#} p<0.1\right)$

\begin{tabular}{ccc}
\hline Contrast & HMI / Urgency & Statistics \\
\hline L1-L2 & w0 & $\mathrm{t}(181.8)=-7.79, \mathrm{p}<0.001$ \\
L1-L3 & w0 & $\mathrm{t}(184.7)=-8.84, \mathrm{p}<0.001$ \\
L1-L4 & w0 & $\mathrm{t}(181.2)=-9.19, \mathrm{p}<0.001$ \\
L1-L2 & w1 & $\mathrm{t}(170.4)=-8.92, \mathrm{p}<0.001$ \\
L1-L3 & w1 & $\mathrm{t}(170.4)=-11.90, \mathrm{p}<0.001$ \\
L1-L4 & w1 & $\mathrm{t}(175.3)=-12.95, \mathrm{p}<0.001$ \\
L2-L3 & w1 & $\mathrm{t}(170.4)=-2.98, \mathrm{p}=0.017$ \\
L2-L4 & w1 & $\mathrm{t}(175.3)=-4.24, \mathrm{p}<0.001$ \\
L1-L2 & w2 & $\mathrm{t}(170.4)=-8.43, \mathrm{p}<0.001$ \\
L1-L3 & w2 & $\mathrm{t}(170.4)=-10.91, \mathrm{p}<0.001$ \\
L1-L4 & w2 & $\mathrm{t}(173.1)=-12.74, \mathrm{p}<0.001$ \\
L2-L3 & w2 & $\mathrm{t}(170.4)=-2.48, \mathrm{p}=0.067$ \\
L2-L4 & w2 & $\mathrm{t}(173.1)=-4.40, \mathrm{p}<0.001$ \\
w0 - w1 & L3 & $\mathrm{t}(142.1)=-3.76, \mathrm{p}=0.001$ \\
w0 - w2 & L3 & $\mathrm{t}(142.1)=-3.06, \mathrm{p}=0.007$ \\
w0 - w1 & L4 & $\mathrm{t}(140.6)=-4.84, \mathrm{p}<0.001$ \\
w0 - w2 & L4 & $\mathrm{t}(143.3)=-4.79, \mathrm{p}<0.001$ \\
\hline
\end{tabular}

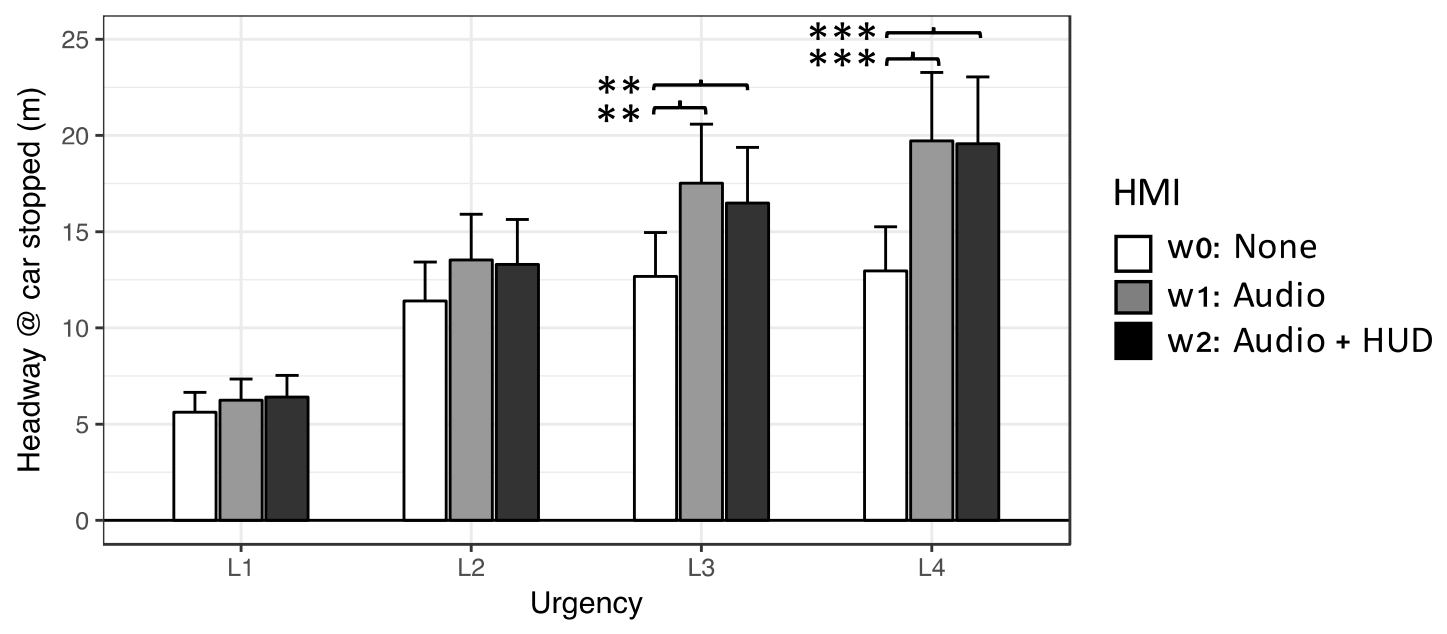

Figure 5: Headway @ Car Stopped by urgency and modality, with 95\% confidence intervals of the means $(* p<0.05, * * p<0.01, * * * p<0.001)$ 


\section{Discussion}

The study explored the effect of urgency (defined here by TTC/false alarm rate) and modality (none, audioonly and audio+HUD) of PCDS alert warnings on driver acceptance and performance associated with an active safety system. In practice, most systems will also attempt to perform autonomous emergency braking (or other primary control interventions) in critical situations. However, first generation systems are still limited in the range of use-cases that can be addressed. Moreover, in the event of a potential collision, all systems will provide a warning to advise drivers of the potential hazard, and encourage them to take evasive action, before the system assumes control. Understanding drivers' behaviour in response to such warnings is therefore important. Building on previous analyses (reported in: $[44,21]$ ), the study employed a generalized mixed effect model using urgency (measured by TTC $=\{2,3,4,5\}$ seconds, with associated false alarm rates) and modality of warning (HMI $=\{$ no, Audio, Audio+HUD $\}$ ) as independent variables. Acceptance is determined based on participants' responses to seven subjective ratings scales, assessing trust, confidence, annoyance, desirability, predictability, reliance and risk (based on existing acceptance/usability assessment protocols [41]), which were completed immediately after each drive. In addition, objective performance was determined using surrogate measures of safety, i.e. the timeto-collision (and/or associated headway) at which drivers' gaze first fixated on the pedestrian at risk, when they first lifted their foot from the accelerator (indicating their first response to the warning), when they put their foot on the brake pedal (indicating the driver's first conscious effort to slow the car), and finally, the headway at which the driver brought the vehicle to a stop. These measures represent the margin of safety between vehicle and the hazard (i.e. vulnerable pedestrian), based on drivers' responses to the warnings.

Immediately apparent is that all acceptance items were generally rated highly at all levels of urgency and for both HMI solutions - above the median scale-point for positive terms, and below this for negative terms indicating a general acceptance of in-vehicle warnings in situations where a potential threat is present (in this case, a pedestrian entering the vehicle's path). On closer inspection, however, there were notable differences in subjective ratings, associated primarily with the time at which warnings were presented (i.e. TTC) (although differences were also indicated regarding the confidence that people placed in the different warning modalities, discussed later). In particular, later warnings (i.e. at TTC of 2.0 seconds), attracted the lowest ratings of trust, confidence, desirability, predictability, reliance and risk (compared to warnings delivered at longer TTCs), even though 2.0-second warnings were arguably the most reliable, i.e. suffered from the lowest occurrence of false alarms. This suggests that drivers perhaps felt that 2.0 -second warnings were issued too late for them to respond successfully (i.e. the pedestrian was already compromised). In such situations, drivers would therefore be reliant upon primary control interventions for collision mitigation/avoidance that would be expected to follow warnings in a real-world PCDS. It also indicates that drivers may be willing to accept the lower perceived reliability (due to errors and imperfection in the system, as well as the dynamic behaviour of pedestrians), that will inevitably accompany the provision of earlier warnings, even at extended headways (up to 5.0-seconds TTC based on the study findings). Of course, this provides additional time for drivers to take corrective action - braking or steering away from the hazard. As such, drivers maintain a sense of control, and this may be an important factor encouraging the acceptance of in-vehicle technology more generally. Even so, there was a tendency for some ratings to reduce at the highest TTCs (in particular, trust and confidence). At this distance, it is possible that drivers were unaware of the specific threat posed by the pedestrian - and therefore questioned how accurate the system's assessment could be, or drivers may have felt that they had sufficient time to respond without the assistance of a PCDS.

Participants also indicated increased confidence in all situations where auditory and visual warnings (presented on the HUD) were combined, although ratings were comparable for other scale items between audio-only and audio+HUD warnings. Providing multi-modal warnings lends itself to redundancy - if the driver fails to see the warning on the HUD, they are still likely to hear the audio, and vice versa, and this may account for increased confidence in this format.

As with the subjective data, there were clear differences evident for the objective performance measures based on urgency, with drivers fixating their gaze on the 'target' pedestrian at longer headway than when earlier warnings were provided. In addition, drivers responded further away (i.e. took their foot off the accelerator, and put their foot on the brake further from the hazard) when warnings were presented at longer TTC. These actions also corresponded with drivers actually bringing their vehicle to a stop further from the pedestrian when warnings were provided at longer TTCs - in particular, the headway at which the vehicle stopped was shorter for warnings delivered at 2 seconds compared to longer TTCs. Differences between having a warning and not having a warning also became more pronounced as TTC increased for all performance measures. This demonstrates the utility and value of providing warnings, as they can help drivers to identify the potential hazard, and enact primary controls; it also demonstrates an inherent trust in the system, in that drivers appeared willing to accept, and act upon, the judgement of the system.

Results indicate that 'modality' made a significant difference for all primary control interventions (foot off accelerator, foot on brake, and bringing the car to a stop), with drivers taking these actions sooner when warnings 
were provided earlier (at TTCs of 4 and 5 seconds). However, these differences were in fact between 'no warning' and 'audio and/or HUD', demonstrating that while the presence of an alert encouraged the driver to respond sooner, the modality in which it is presented appeared to make little difference. Interestingly, there were no equivalent benefits in localising the target pedestrian in situations when a warning was provided - this is particularly noteworthy given that part of the intention of providing conformal warnings was to guide attention towards the hazard.

In situations where a warning was provided, modality (i.e. audio-only or audio+HUD alerts) only influenced 'foot-on-brake' at 5.0-second TTC, with combined audio and HUD warnings encouraging drivers to apply the brake further from the hazard. It is therefore particularly interesting to note that drivers claimed greater confidence when warnings were also provided on the HUD (in addition to the auditory alarm), given that there were very limited performance gains in doing so.

Although we are confident of the value and contribution of this work, we acknowledge several limitations in our approach. Firstly, to define 'urgency', we assumed that false alarm rate and TTC are closely related and increase together at a linear rate. While there is strong evidence to support this assumption (e.g. [40]), it is nevertheless unlikely that this relationship is completely linear. Modelling system performance in this manner may therefore be an over-simplification. It also means that it is impossible to differentiate the effects of TTC and false alarm rate in our results, although these factors are undeniably linked. In practice, this approach was chosen to provide a more ecologically-valid experience of 'urgency' - it would be unrealistic to expect perfect human intent prediction five seconds into the future. Equally, it would be unusual to experience very high levels of inaccuracy at short TTCs where intent modelling is likely to be more robust (particularly with commercially available systems).

In addition, we limited our investigation to false positive alarms, which were in effect due to an imprecision of the PCDS - a useful warning was given as a pedestrian hazard did exist, and still had the potential to enter the roadway. This arguably mitigates the previous concern to some extent, although we recognise that in practice, there are many other factors that are likely to influence the reliability and capability of such systems. For example, in a real-world situation, subtle cues, such as head-movements, eye-contact and other postural changes, would likely indicate a pedestrian's intent to enter the roadway, or conversely demonstrate that they were about to stop. Such behaviour was notably absent from the simulated environment (due to limitations in the fidelity/functionality of the simulation software). Instead, all pedestrians appeared visually similar to drivers, with the exception that some continued to walk into the roadway, and others did not, and this may have affected drivers' behaviour and responses.

Finally, notwithstanding the aforementioned concerns, it is worth bearing in mind that exposing drivers to regular and repeated warnings meant that they may have become hyper-vigilant to potential pedestrian threats. Although we attempted to address this concern as much as practicable by counterbalancing exposure, there will be inevitable influences on drivers' behaviour caused by the rapid, repeated exposure to potential threats, and thus all results should be considered as relative, as indeed they are presented.

\section{Conclusions}

Warnings are a common component of pedestrian safety systems, intending to make drivers aware of a potential hazard, encouraging them to initiate an evasive manoeuvre, or preparing them for an emergency control intervention (such as steering or braking). Thus, understanding the effect of urgency (i.e. when alarms are declared) and modality (how they are presented) on driver acceptance and performance is important. In the study, warnings were generally well received, inspiring positive ratings of 'acceptance' even at extended TTCs, and despite relatively high false alarm rates. In addition, earlier warnings helped drivers localise the potential threat sooner, and encouraged them to begin evasive braking manoeuvres further from the pedestrian. There was, however, little differentiation in drivers' performance between audio and audio/visual presentations, and modality also appeared to have negligible overall effect on generalised 'acceptance' ratings. An exception to this was drivers' confidence, which increased with multi-modal presentation, suggesting redundancy in modality improved self-efficacy. In addition, delaying warnings (i.e. presenting them at shorter TTCs) detrimented trust, confidence, desirability, predictability, reliance and risk, even though later warnings invited fewer false activations. Ultimately, we can conclude that providing earlier warnings has the potential to increase the margin of safety between car and pedestrian, without necessarily detrimenting acceptance. Moreover, multi-modal warnings are likely to increase confidence even though these do not necessarily lead to improvements in drivers' performance. 


\section{Acknowledgements}

The research received funding from the European Community's Eighth Framework Program (Horizon2020) (agreement no. 634149: Proactive Safety for Pedestrians and Cyclists (PROSPECT) project), and the NSF-funded International Research Experience for Students (IRES) program between Virginia Tech University and the University of Nottingham (NSF award: 1261162).

\section{References}

[1] World Health Organization, "Global status report on road safety,” 2015.

[2] K. J. Clifton, C. V. Burnier and G. Akar, "Severity of injury resulting from pedestrian-vehicle crashes: What can we learn from examining the built environment?," Transportation research part D: transport and environment, vol. 14, pp. 425-436, 2009.

[3] L. F. Beck, A. M. Dellinger and M. E. O'neil, "Motor vehicle crash injury rates by mode of travel, United States: using exposure-based methods to quantify differences," American Journal of Epidemiology, vol. 166, pp. 212-218, 2007.

[4] F. Graves, D. Lloyd, D. Wilson, D. Mais, W. Deda and A. Bhagat, "Reported Road Casualties Great Britain: 2013 Annual Report,” 2014.

[5] M. Yanagisawa, E. Swanson and W. G. Najm, "Target Crashes and Safety Benefits Estimation Methodology for Pedestrian Crash Avoidance/Mitigation Systems,” 2014.

[6] PROSPECT (Proactive Safety for Pedestrians and Cyclists), "Deliverable D2.1: Accident Analysis, Naturalistic Observations and Project Implications," 2016.

[7] EUROPEAN NEW CAR ASSESSMENT PROGRAMME (Euro NCAP), "TEST PROTOCOL AEB VRU systems (version 2.0),” 2017.

[8] PROSPECT (Proactive Safety for Pedestrians and Cyclists), "Deliverable D5.2: Advanced HMI and vehicle control concepts for VRU active safety," 2017.

[9] A. Silla, P. Rama, L. Leden, M. van Noort, J. de Kruijff, D. Bell, A. Morris, G. Hancox and J. Scholliers, "Are intelligent transport systems effective in improving the safety of vulnerable road users?," 2015.

[10] S. Zhang, R. Benenson, M. Omran, J. Hosang and B. Schiele, "How Far are We from Solving Pedestrian Detection?," arXiv preprint arXiv:1602.01237, 2016.

[11] R. Parasuraman and V. Riley, "Human and automation: use, misuse, disuse, abuse," Human Factors, vol. 39, no. 2, pp. 230-253, 1997.

[12] V. Banks and N. Stanton, "Keep the driver in control: automating automobiles of the future," Applied Ergonomics, vol. 53, no. B, pp. 389-395, 2015.

[13] D. Norman, The design of future things, New York: Basic Books, 2007.

[14] C. Baldwin and B. Lewis, "Perceived urgency mapping across modalities within a driving context," Applied Ergonomics, vol. 45, pp. 1270-1277, 2014.

[15] E. Adell, Driver experience and acceptance of driver support systems: a case of speed adaptation, 2009.

[16] M. Ghazizadeh, J. Lee and L. Boyle, "Extending the Technology Acceptance Model to assess automation," Cognition, Technology \& Work, vol. 14, no. 1, pp. 39-49, 2012.

[17] J. Schade and M. Baum, "Reactance or acceptance? Reactions towards the introduction of road pricing," Transportation Research Part A: Policy and Practice, vol. 41, no. 1, pp. 41-48, 2007.

[18] K. Ausserer and R. Risser, "Intelligent transport systems and services-chances and risks," in ICTCTworkshop, 2005.

[19] T. Sheridan, "Risk, human error, and system resilience: fundamental ideas," Human Factors: The Journal of the Human Factors and Ergonomics Society, vol. 50, no. 3, pp. 418-426, 2008. 
[20] J. Källhammer and K. Smith, "Assessing contextual factors that influence acceptance of pedestrian alerts by a night vision system," Human Factors: The Journal of the Human Factors and Ergonomics Society, vol. 54, no. 4, pp. 654-662, 2012.

[21] C. Merenda, H. Kim, S. Leong, J. Gabbard, G. Burnett and D. Large, "Did you see me? Assessing Real vs. Perceived Driving Gains across Multi-modality Pedestrian Alert Systems," in The 9th International ACM Conference on Automotive User Interfaces and Interactive Vehicular Applications (AutoUI2017), Oldenburg, Germany, 2017.

[22] J. De Boer, A. Chaziris, J. Vreeswijk, J. Bie and B. Van Arem, “The accuracy and timing of pedestrian warnings at intersections: The acceptance from drivers and their preferences," in 13th International IEEE Conference on Intelligent Transportation Systems (ITSC), , 2010.

[23] J. Lee, D. McGehee, T. Brown and M. Reyes, "Collision warning timing, driver distraction, and driver response to imminent rear-end collisions in a high-fidelity driving simulator," Human Factors: The Journal of the Human Factors and Ergonomics Society, vol. 44, no. 2, pp. 314-334, 2002.

[24] J. Lee and B. Kantowitz, "Perceptual and cognitive aspects of intelligent transportation systems," Human factors in intelligent transportation systems, 1998.

[25] J. D. Lee and K. A. See, "Trust in automation: designing for appropriate reliance," Human Factors, vol. 46, no. 1, pp. 50-80, 2004.

[26] L. Bainbridge, "Ironies of automation,” Automatica, vol. 19, no. 6, pp. 775-779, 1983.

[27] A. Löcken, A. Unni, H. Müller and S. Boll, "The car that cares: introducing an in-vehicle ambient display to reduce cognitive load," in Automotive User Interfaces and Interactive Vehicular Applications, Eindhoven, The Netherlands, 2013.

[28] A. Meschtscherjakov, C. Döttlinger, C. Rödel and M. Tscheligi, "ChaseLight: ambient LED strips to control driving speed," in Automotive User Interfaces and Interactive Vehicular Applications, Nottingham, UK, 2015.

[29] G. Burnett, "A road-based evaluation of a head-up display for presenting navigation information," in Proceedings of the HCI international conference, Crete, 2003.

[30] D. Foyle, B. Hooey, J. Wilson and W. Johnson, "HUD symbology for surface operations: command guidance vs. situation guidance formats," SAE Transactions: Journal of Aerospace, vol. 111, pp. 647-658, 2002.

[31] Y.-C. Liu and M.-H. Wen, "Comparison of head-up display (HUD) vs. head-down display (HDD): driving performance of commercial vehicle operators in Taiwan," International Journal of HumanComputer Studies, vol. 61, no. 5, pp. 679-697, 2004.

[32] H. Kim, A. Miranda Anon, T. Misu, N. Li, A. Tawari and K. Fujimura, "Look at Me: Augmented Reality Pedestrian Warning System Using an In-Vehicle Volumetric Head Up Display," in Proceedings of the 21 st International Conference on Intelligent User Interfaces, 2016.

[33] R. Eyraud, E. Zibetti and T. Baccino, "Allocation of visual attention while driving with simulated augmented reality," Transportation research part F: traffic psychology and behaviour, vol. 32, pp. 46-55, 2015.

[34] P. Rane, H. Kim, J. Marcano and J. Gabbard, "Virtual road signs: Augmented reality driving aid for novice drivers," in Proceedings of the Human Factors and Ergonomics Society Annual Meeting, Los Angeles, 2016.

[35] H. Kim, J. Isleib and J. Gabbard, "Virtual Shadow: Making Cross Traffic Dynamics Visible through Augmented Reality Head Up Display," in Proceedings of the Human Factors and Ergonomics Society Annual Meeting, Los Angeles, 2016.

[36] T. Sayer, J. Sayer and J. Devonshire, “Assessment of a driver interface for lateral drift and curve speed warning systems: mixed results for auditory and haptic warnings," in Proceedings of Driving Assessment, 2005. 
[37] M. Blanco, J. Atwood, H. Vasquez, T. Trimble, V. Fitchett, V. Radlbeck, G. Fitch, S. Russell, C. Green, B. Cullinane and J. Morgan, Human factors evaluation of level 2 and level 3 automated driving concepts, Washington, DC, 2015.

[38] C. Ho and C. Spence, The multisensory driver: implications for ergonomic car interface design, Aldershot: Ashgate, 2008.

[39] R. Gray, C. Spence, C. Ho and H. Tan, "Efficient multimodal cuing of spatial attention," Proceedings of the IEEE, vol. 10, pp. 2113-2122, 2013.

[40] C. Keller and D. Gavrila, "Will the pedestrian cross? a study on pedestrian path prediction," IEEE Transactions on Intelligent Transportation Systems, vol. 15, no. 2, pp. 494-506, 2014.

[41] J. Brooke, “ SUS-A quick and dirty usability scale," Usability evaluation in industry, vol. 189, no. 194, pp. 4-7, 1996.

[42] J. L. Campbell, C. M. Richard, J. L. Brown and M. McCallum, "Crash warning system interfaces: human factors insights and lessons learned," US Department of Transportation, National Highway Traffic Safety Administration, 2007.

[43] N. Reader, "Development and validation of functional definitions and evaluation procedures for collision warning/avoidance systems," 1999.

[44] D. R. Large, C. Harvey, G. Burnett, C. Merenda, S. Leong and Gabbard, "Exploring the Relationship Between False Alarms and Driver Acceptance of a Pedestrian Alert System During Simulated Driving," in Road Safety and Simulation International Conference, The Hague, Netherlands, 2017. 